Article

\title{
Serum Urokinase-Type Plasminogen Activator Receptor Does Not Outperform C-Reactive Protein and Procalcitonin as an Early Marker of Severity of Acute Pancreatitis
}

\author{
Witold Kolber ${ }^{1}$, Beata Kuśnierz-Cabala ${ }^{2, *}$, Paulina Dumnicka ${ }^{3}$, Małgorzata Maraj ${ }^{4}$, \\ Małgorzata Mazur-Laskowska ${ }^{5}$, Michał Pędziwiatr ${ }^{6}$ and Piotr Ceranowicz ${ }^{4}$ \\ 1 Department of Surgery, Complex of Health Care Centers in Wadowice, Karmelicka 5 St., 34-100 Wadowice, \\ Poland; wkolber@wp.pl \\ 2 Department of Diagnostics, Chair of Clinical Biochemistry, Faculty of Medicine, Jagiellonian University \\ Medical College, Kopernika 15A St., 31-501 Krakow, Poland \\ 3 Department of Medical Diagnostics, Faculty of Pharmacy, Jagiellonian University Medical College, \\ Medyczna 9 St., 30-688 Krakow, Poland; paulina.dumnicka@uj.edu.pl \\ 4 Department of Physiology, Faculty of Medicine, Jagiellonian University Medical College, Grzegorzecka 16 \\ St., 31-531 Krakow, Poland; malgorzata.maraj@gmail.com (M.M.); mpcerano@cyf-kr.edu.pl (P.C.) \\ 5 Diagnostics Department of University Hospital in Krakow, Kopernika 15B St., 31-501 Krakow, Poland; \\ mbmazur@cyf-kr.edu.pl \\ 6 2nd Department of Surgery, Faculty of Medicine, Jagiellonian University Medical College, Kopernika 21 St., \\ 31-501 Krakow, Poland; mpedziwiatr@gmail.com \\ * Correspondence: mbkusnie@cyf-kr.edu.pl; Tel./Fax: +48-12-424-8371
}

Received: 8 August 2018; Accepted: 19 September 2018; Published: 27 September 2018

check for updates

\begin{abstract}
Severe acute pancreatitis (SAP) concerns 10-20\% of acute pancreatitis (AP) patients and is associated with a poor prognosis and high mortality. An early prognosis of the unfavorable outcome, transfer to an intensive care unit (ICU) and the introduction of an adequate treatment are crucial for patients' survival. Recently, the elevated circulating urokinase-type plasminogen activator receptor (uPAR) has been reported to predict SAP with a high diagnostic accuracy among patients in a tertiary center. The aim of the study was to compare the diagnostic utility of uPAR and other inflammatory markers as the predictors of the unfavorable course of AP in patients admitted to a secondary care hospital within the first $24 \mathrm{~h}$ of the onset of AP. The study included 95 patients, eight with a SAP diagnosis. Serum uPAR was measured on admission and in the two subsequent days. On admission, uPAR significantly predicted organ failure, acute cardiovascular failure, acute kidney injury, the need for intensive care, and death. The diagnostic accuracy of the admission UPAR for the prediction of SAP, organ failure, and ICU transfer or death was low to moderate and did not differ significantly from the diagnostic accuracy of interleukin-6, C-reactive protein, procalcitonin, D-dimer and soluble fms-like tyrosine kinase-1. In the secondary care hospital, where most patients with AP are initially admitted, uPAR measurements did not prove better than the currently used markers.
\end{abstract}

Keywords: urokinase-type plasminogen activator receptor; interleukin 6; early prediction of acute pancreatitis severity; acute kidney injury; liver failure

\section{Introduction}

Acute pancreatitis (AP) is one of the most common acute digestive tract diseases and, despite significant medical advancement in the last decade, it still poses a risk of life-threatening 
complications. In case of severe acute pancreatitis (SAP), mortality can reach $20-30 \%$ [1-3]. Recent studies indicate that in consequence of the systemic inflammatory response syndrome (SIRS) and multiple organ dysfunction syndrome (MODS) nearly half of deaths occur within the first week of AP [1,4,5]. These findings have been reflected in the 2012 revised Atlanta classification [6], which defines the early and the late phases of the disease. The early phase covers the period of one week from the onset of symptoms and is followed by the late phase lasting weeks or even months. The first 48 $\mathrm{h}$ are important for the further course of $\mathrm{AP}$ as adequate clinical management (including intensive fluid resuscitation) during the so-called therapeutic window can reduce the risk of complications $[1,3,4,7]$. This is especially important for patients developing MODS who should be referred to an intensive care unit (ICU) [7]. The diagnostic process to assess AP severity should take place in the first $48 \mathrm{~h}$ from the onset of symptoms. At present, it involves clinical assessment, imaging tests, multi-variable predictive scores (Ranson, APACHE II, Glasgow, BISAP) and laboratory testing [1,3,7,8]. Unfortunately, none of the above diagnostic tools appears flawless.

The laboratory tests that have been proposed for the assessment of AP severity include proinflammatory [tumor necrosis factor- $\alpha$ (TNF- $\alpha$ ), interleukins (IL): IL-6, IL-1 $\beta$, IL-18, IL-8] and anti-inflammatory (IL-4, IL-10, IL-1 receptor antagonist) mediators, soluble receptors (soluble TNF- $\alpha$ receptor II), microRNAs, adhesive molecules (soluble intercellular adhesion molecule 1), growth factors (hepatocyte growth factor, tumor growth factor- $\beta 1$ ), procalcitonin, and acute phase proteins [C-reactive protein (CRP), serum amyloid A] [5,9-11]. However, many of the above markers can only be measured with enzyme-linked immunoassays rarely conducted in real time because of long procedures and high costs when not applied in series. At present, no single laboratory marker can be recommended for AP severity stratification, although in the clinical practice CRP measurements are often used [4]. Moreover, IL-6 and procalcitonin can be useful, as patients with SAP present with clearly higher concentrations in the first $48 \mathrm{~h}$ from the onset of symptoms: $\geq 300 \mathrm{pg} / \mathrm{mL}$ [12] and $\geq 3.3 \mathrm{ng} / \mathrm{mL}$, respectively $[9,10,13]$.

Patients with SIRS of either infectious or non-infectious origin present a severe endothelial dysfunction and hypercoagulability [14-16]. Endothelial dysfunction, together with tissue hypoperfusion, lead to gradual progression of organ failure $[5,14,17]$. The process is accompanied by the activation of coagulation and fibrinolysis, reflected in altered results of laboratory coagulation tests, e.g., high D-dimer concentrations $[16,18]$.

One of the earliest fibrinolysis mediators is the urokinase-type plasminogen activator (uPA) [17]. Its ability to convert plasminogen into plasmin is potentiated by binding to the UPA receptor (uPAR, CD87) expressed on the endothelial cells, monocytes, macrophages, T-cells and granulocytes [17,19-21]. Besides being involved in fibrinolysis, UPAR assists in cell adhesion, migration, chemotaxis, immune activation, and tissue reconstruction [22,23]. The protein is bound to cell membranes by glycosylphosphatidylinositol anchor and may be shed or released into body fluids [23]. Soluble uPAR is present in plasma or serum, urine and cerebrospinal fluid [20-23]. Increased serum uPAR has been associated with various inflammatory states, particularly with sepsis and non-septic SIRS [17,20-22]. The correlation with severity of organ failure and mortality, together with the relative stability of plasma or serum concentrations, make uPAR a promising clinical biomarker in patients with SIRS [20,22,24,25], but so far has not been extensively studied in AP. Nonetheless, in 2017, Lipinski et al. [26] showed a very high diagnostic accuracy of plasma uPAR for the prediction of SAP and fatal AP among patients of a tertiary referral hospital.

The aim of our study was to assess the diagnostic usefulness of UPAR in the prediction of severe course of AP in a secondary care hospital setting, where most patients with AP are initially admitted. We were interested to know whether uPAR testing may help in the early (preferably within the first $48 \mathrm{~h}$ from the onset of AP symptoms) prediction of organ failure and, thus, the need for ICU transfer, and whether in this regard uPAR outperforms other single laboratory markers associated with AP severity. 


\section{Materials and Methods}

The prospective observational study was designed to test whether the concentrations of uPAR measured in serum on the first days of AP allow the prediction of disease severity assessed during the hospital stay, and to compare UPAR with the other single laboratory tests used to predict AP severity. The study included adult patients admitted with AP, treated at the Surgery Ward, Complex of Health Care Centers in Wadowice, Poland (a secondary care hospital). Consecutive patients with a diagnosis of AP who were admitted to the Ward between March 2014 and December 2015 were assessed regarding eligibility for the study. Only patients with symptoms of AP lasting up to $24 \mathrm{~h}$ before hospital admission were included in the study. The exclusion criteria were chronic pancreatitis, known malignancy, and chronic liver disease. The patients provided written informed consent for the study. The study included patients who were able to provide an informed consent within a day of admission. The patients included in the study were asked to give three blood samples for laboratory tests, one sample on each of the first three days of the hospital stay. The demographic and clinical data were collected, including the etiology of AP, data on complications, severity and treatment of AP during the hospital stay. The main outcome variables were the organ failure during the first week of the hospital stay, the severity of AP defined according to the 2012 Atlanta classification (based on the entire course of the hospital stay) [6], and the ICU transfer or death during the hospital stay. The study protocol was approved by the Bioethics Committee of the Beskidy Medical Chamber (approval number 2014/02/06/1 issued on 6 February 2014).

The diagnosis of AP was based on the 2012 revised Atlanta classification [6], when at least two out of three of the following criteria were positive, i.e., typical clinical presentation of AP, serum amylase or lipase activity three times the upper reference value, and typical findings in dynamic computed tomography or magnetic resonance imaging or ultrasonography. The severity of AP was classified according to the 2012 Atlanta classification [6] as mild acute pancreatitis (MAP), moderately severe acute pancreatitis (MSAP) or severe acute pancreatitis (SAP). Organ failure was identified using the Modified Marshall Scoring System (MMSS) [6]. The diagnosis of acute kidney injury (AKI) was based on the Kidney Disease Improving Global Outcomes (KDIGO) criteria [27]. The diagnosis of acute respiratory distress syndrome (ARDS) was based on the Berlin criteria [28].

On admission (day 1), and on the two following days (day 2 and 3), blood samples were obtained from patients for routine blood tests and for measurements of uPAR, IL-6 and soluble fms-like tyrosine kinase-1 (sFlt-1). The routine blood tests included complete blood cell count, serum concentrations of urea, creatinine, bilirubin, CRP and procalcitonin, serum activities of amylase, lactate dehydrogenase (LDH), aspartate and alanine aminotransferases (AST, ALT), and plasma concentrations of D-dimer. The routine tests were conducted on the day of blood collection in the Central Laboratory, Complex of Health Care Centers in Wadowice, Poland using automatic analyzers: Sysmex XN (Sysmex Corporation, Cobe, Japan) for the blood counts, Cobas E411 (Roche Diagnostics, Mannheim, Germany) and Vitros 5600 (Ortho Clinical Diagnostics, Raritan, NJ, USA) for biochemistry and immunochemistry, and Coag XL (Diagon, Budapest, Hungary) for the coagulation testing. The performance of routine laboratory tests was assessed with daily internal quality control and regular external quality control (including Centre for Quality Assessment in Laboratory Medicine in Poland and Randox International Quality Assessment Scheme, RIQAS), in line with good laboratory practice. Additional serum samples for IL-6, uPAR and sFlt- 1 assessment were aliquoted and frozen in $-80^{\circ} \mathrm{C}$. IL-6 and sFlt-1 were measured by electrochemiluminescence immunoassay (ECLIA) on Cobas 8000 analyzer (Roche Diagnostics, Mannheim, Germany) in the Diagnostic Department of University Hospital in Krakow, Poland. The minimum detectable doses were $1.5 \mathrm{pg} / \mathrm{mL}$ for IL-6 and $10 \mathrm{pg} / \mathrm{mL}$ for sFlt-1. The tests were calibrated according to the manufacturer's instruction, the measurements in patients' samples were run in series and were preceded by controls (PreciControl Multimaker, Roche, Mannheim, Germany) on two levels. For the IL- 6 test, the intraassay precision was $\leq 6.0 \%$ and the interassay precision was $\leq 8.5 \%$, and for the sFlt- 1 test, the intraassay precision was $\leq 3.9$ and the interassay precision was $\leq 5.6 \%$, as reported by the manufacturer of the tests. Serum uPAR 
was measured with enzyme-linked immunoassay, using Quantikine Human uPAR Immunoassay reagent kit (R\&D Systems, Minneapolis, MN, USA) in the Department of Diagnostics, Chair of Clinical Biochemistry at Jagiellonian University, Krakow, Poland. The minimum detectable dose of uPAR was $0.033 \mathrm{ng} / \mathrm{mL}$, the reference range provided by the manufacturer was $1.195-4.415 \mathrm{ng} / \mathrm{mL}$. Patients' samples were tested for UPAR in duplicates, and in series; each run was calibrated according to the manufacturer's instructions. The intraassay precision for the test was $\leq 7.5 \%$ and the interassay precision $\leq 5.6 \%$.

Nominal data were reported as number (percentage of the group). Quantitative data were reported as mean and standard deviation (SD) or median, lower and upper quartiles (Q1; Q3), depending on normality of each variable's distribution (as assessed with Shapiro-Wilk's test). The contingency tables were analyzed with Pearson's chi-squared test. In case of three groups, the whole contingency table was analyzed first, and then the pairwise comparisons were done using Pearson chi-squared test with Bonferroni correction. Due to the non-normal distribution of most quantitative variables, Kruskal-Wallis's analysis of variance (with post-hoc comparisons using Siegel and Castellan method) was applied when three groups were compared and Mann-Whitney's test when two groups were compared. Spearman's rank order coefficient was used to assess correlations. Simple and multiple logistic regression was used to assess UPAR as a predictor of unfavorable course of AP. Receiver operating characteristic (ROC) curves were used to evaluate the diagnostic accuracy of the studied laboratory tests; the cut-off values were selected by maximizing the Youden index. The statistical tests were two-tailed, and the results were considered significant at $p<0.05$. Statistica 12 software (Statsoft, Tulsa, OK, USA) was used for computations.

\section{Results}

The study included 95 patients with AP, 30 women, 65 men, with a mean (SD) age of 48 (16) years. According to the 2012 Atlanta criteria, 29 patients (31\%) were diagnosed with MAP, 58 (61\%) with MSAP and $8(8 \%)$ with SAP. MAP, MSAP and SAP patients did not differ significantly in terms of age, sex, percentage affected with comorbidities, and AP etiology (Table 1). Average Ranson's score was higher among patients with MSAP and SAP, while SIRS was common among both MSAP and SAP patients. The patients' treatment (the need for surgery, nutritional support and ICU transfer) reflected the severity of AP. Four patients (4\%) died-one in the early and three in the late phase of AP (Table 1).

On admission, the patients with SAP were characterized with significantly higher concentrations of urea, creatinine, glucose and IL-6 (Table 2). Other studied laboratory markers did not differ significantly between the MAP, MSAP and SAP patients on admission, although the median concentrations of inflammatory markers: Serum uPAR, CRP, procalcitonin and plasma D-dimer, as well as the median numbers of leukocytes were relatively high among patients with SAP. This was also observed in the case of endothelial dysfunction marker, i.e., sFlt-1 (Table 2).

Serum uPAR concentrations were significantly higher in women as compared to men, starting from the day of admission (Figure 1). No sex-related differences were observed for any other inflammatory markers (IL-6: $p>0.5$; procalcitonin: $p>0.2$; D-dimer: $p>0.1$; white blood cells: $p>0.1$; admission and day 3 CRP: $p>0.1$ ), except for the day 2, when CRP was higher in men (median 315 in men versus $185 \mathrm{mg} / \mathrm{L}$ in women; $p=0.011$ ). Also, no significant differences were observed between men and women regarding the severity of AP as defined according to the Atlanta classification (Table 1). Serum uPAR concentrations did not differ between patients with AP of various etiology ( $p=0.7$ on admission and day 2; $p=0.2$ on day 3 ). No correlations were observed between uPAR and age. Patients with comorbid conditions (mainly ischemic heart disease) tended to present higher concentrations of uPAR, however, the difference was only significant on day 2 of the study: Median (Q1; Q3) was 3.77 $(2.97 ; 5.03) \mathrm{ng} / \mathrm{mL}$ in those affected by comorbidities versus $2.93(2.54 ; 3.88) \mathrm{ng} / \mathrm{mL}$ in the patients without comorbidities $(p=0.023)$. 
Table 1. Clinical characteristics of the study group according to the severity of acute pancreatitis (AP).

\begin{tabular}{|c|c|c|c|c|}
\hline Characteristic & MAP $(n=29)$ & MSAP $(n=58)$ & SAP $(n=8)$ & $p$-Value \\
\hline Male sex, $n(\%)$ & $17(59)$ & $41(71)$ & $7(88)$ & 0.2 \\
\hline Mean age (SD), years & $43(16)$ & $50(16)$ & $51(20)$ & 0.1 \\
\hline Pre-existing comorbidities, $n(\%)$ & $10(34)$ & $27(47)$ & $5(62)$ & 0.3 \\
\hline Cardiac diseases, $n(\%)$ & $5(17)$ & $20(34)$ & $5(62)$ & \\
\hline Diabetes, $n(\%)$ & 0 & $6(10)$ & $2(25)$ & \\
\hline Dyslipidemia, $n(\%)$ & $1(3)$ & $2(3)$ & 0 & \\
\hline Chronic kidney disease, $\mathrm{n}(\%)$ & 0 & $2(3)$ & 0 & \\
\hline Liver disease, $n(\%)$ & $1(3)$ & $2(3)$ & 0 & \\
\hline Other comorbidities, $n(\%)$ & $3(10)$ & 0 & 0 & \\
\hline Etiology & & & & 0.1 \\
\hline Biliary, $n(\%)$ & $9(31)$ & $17(29)$ & $1(12)$ & \\
\hline Alcoholic, $n(\%)$ & $12(41)$ & $11(19)$ & $6(75)$ & \\
\hline Hipertriglyceridemia, $n(\%)$ & $1(3)$ & $4(7)$ & 0 & \\
\hline Other/idiopathic, $n(\%)$ & $7(24)$ & $26(45)$ & $1(12)$ & \\
\hline Median Ranson score (Q1; Q3), points & $2(1 ; 3)$ & $3(3 ; 4)$ & $6(4 ; 7)$ & $<0.001^{\mathrm{a}, \mathrm{b}, \mathrm{c}}$ \\
\hline Median duration of hospital stay(Q1; Q3), days & $10(7 ; 12)$ & $14(10 ; 16)$ & $26(13 ; 41)$ & $0.001^{\mathrm{a}, \mathrm{c}}$ \\
\hline SIRS in first $24 \mathrm{~h}, n(\%)$ & $18(62)$ & $49(84)$ & $7(88)$ & $0.047^{c}$ \\
\hline Early/late mortality, $n(\%)$ & 0 & $0 / 2(3)$ & $1(12) / 1(12)$ & $0.006^{a, b}$ \\
\hline Therapeutic ERCP, $n(\%)$ & 0 & $3(5)$ & $2(25)$ & $0.020^{\mathrm{a}, \mathrm{b}}$ \\
\hline Surgery, $n(\%)$ & 0 & $3(5)$ & $4(50)$ & $<0.001^{\mathrm{a}, \mathrm{b}}$ \\
\hline Enteral feeding via nasojejunal tube, $n(\%)$ & 0 & $4(7)$ & $6(75)$ & $<0.001^{\mathrm{a}, \mathrm{b}}$ \\
\hline Parenteral feeding, $n(\%)$ & 0 & $1(2)$ & $2(25)$ & $0.001^{\mathrm{a}, \mathrm{b}}$ \\
\hline Transfer to ICU, $n(\%)$ & 0 & $2(3)$ & $5(62)$ & $<0.001^{\mathrm{a}, \mathrm{b}}$ \\
\hline
\end{tabular}

ERCP, endoscopic retrograde cholangiopancreatography; ICU, intensive care unit; MAP, mild acute pancreatitis; MSAP, moderately severe acute pancreatitis; $n$, number of patients; SAP, severe acute pancreatitis; $\mathrm{SD}$, standard deviation; SIRS, systemic inflammatory response syndrome; Q1, lower quartile; Q3, upper quartile; $p$-value is reported for overall comparison between three groups (in Pearson chi-squared test or Kruskal-Wallis ANOVA), the letters in superscript indicate the results of post-hoc tests: ${ }^{a}$ significant difference between the MAP and SAP groups in post-hoc comparison; ${ }^{b}$ significant difference between the MSAP and SAP groups in post-hoc comparison; ${ }^{c}$ significant difference between the MAP and MSAP groups in post-hoc comparison.

Table 2. The results of laboratory tests on admission according to the AP severity. Data are shown as mean (SD) or median (Q1; Q3).

\begin{tabular}{ccccc}
\hline Variable & MAP $(\boldsymbol{n}=\mathbf{2 9 )}$ & MSAP $(\boldsymbol{n}=\mathbf{5 8})$ & SAP $(\boldsymbol{n}=\mathbf{8})$ & $p$-Value \\
\hline Hematocrit, $\%$ & $42(5)$ & $43(6)$ & $46(7)$ & 0.4 \\
Albumin, $\mathrm{g} / \mathrm{L}$ & $38(7)$ & $35(6)$ & $36(8)$ & 0.6 \\
Total calcium, mmol/L & $2.13(0.23)$ & $2.15(0.19)$ & $1.92(0.48)$ & 0.5 \\
Glucose, $\mathrm{mmol} / \mathrm{L}$ & $6.44(5.61 ; 7.67)$ & $8.17(6.78 ; 9.33)$ & $7.92(7.22 ; 10.64)$ & $0.002^{\mathrm{a}, \mathrm{c}}$ \\
Urea, $\mathrm{mmol} / \mathrm{L}$ & $3.67(2.83 ; 6.00)$ & $4.67(3.50 ; 6.00)$ & $6.67(5.00 ; 13.00)$ & $0.015^{\mathrm{a}}$ \\
Creatinine, $\mu \mathrm{mol} / \mathrm{L}$ & $65.4(59.2 ; 80.4)$ & $69.8(60.1 ; 87.5)$ & $92.4(75.6 ; 171.1)$ & $0.033^{\text {a }}$ \\
Bilirubin, $\mu \mathrm{mol} / \mathrm{L}$ & $23.4(13.5 ; 38.5)$ & $27.2(13.8 ; 53.3)$ & $29.1(16.2 ; 36.9)$ & 0.7 \\
AST, U/L & $59(33 ; 209)$ & $116(55 ; 202)$ & $122(87 ; 166)$ & 0.3 \\
ALT, U/L & $62(43 ; 174)$ & $133(54 ; 299)$ & $85(49 ; 158)$ & 0.3 \\
LDH, U/L & $553(488 ; 810)$ & $636(507 ; 850)$ & $1013(737 ; 1294)$ & 0.1 \\
WBC, $\times 10^{3} / \mu \mathrm{L}$ & $12.4(9.5 ; 15.2)$ & $13.1(10.4 ; 16.2)$ & $17.1(10.3 ; 23.3)$ & 0.4 \\
Platelet count, $\times 10^{3} / \mu \mathrm{L}$ & $199(176 ; 231)$ & $218(165 ; 279)$ & $227(162 ; 292)$ & 0.8 \\
CRP, $\mathrm{mg} / \mathrm{L}$ & $22.7(5.3 ; 132.4)$ & $25.4(11.9 ; 174.7)$ & $129.6(17.4 ; 316.7)$ & 0.4 \\
D-dimer, $\mathrm{mg} / \mathrm{L}$ & $1.49(0.85 ; 2.19)$ & $1.90(1.00 ; 3.41)$ & $2.76(1.20 ; 3.39)$ & 0.2 \\
Procalcitonin, $\mathrm{ng} / \mathrm{mL}$ & $0.10(0.05 ; 0.55)$ & $0.17(0.10 ; 0.36)$ & $0.61(0.14 ; 1.03)$ & 0.1 \\
uPAR, ng/mL & $3.92(2.86 ; 5.02)$ & $3.81(2.98 ; 5.10)$ & $5.00(3.41 ; 6.74)$ & 0.4 \\
Interleukin $6, \mathrm{pg} / \mathrm{mL}$ & $64.7(14.8 ; 95.7)$ & $78.9(27.8 ; 163.0)$ & $210.7(73.1 ; 21.5)$ & $0.037^{\mathrm{a}}$ \\
sFlt-1, $\mathrm{pg} / \mathrm{mL}$ & $129(119 ; 169)$ & $140(112 ; 154)$ & $191(155 ; 536)$ & 0.1 \\
\hline
\end{tabular}

ALT, alanine aminotransferase; AST, aspartate aminotransferase; CRP, C-reactive protein; LDH, lactate dehydrogenase; MAP, mild acute pancreatitis, MSAP, moderately severe acute pancreatitis; SAP, severe acute pancreatitis; sFlt-1, soluble fms-like tyrosine kinase-1; uPAR, urokinase-type plasminogen activator receptor; WBC, white blood cells; $p$-value is reported for overall comparison between three groups (in Kruskal-Wallis ANOVA), the letters in superscript indicate the results of post-hoc tests: ${ }^{\text {a }}$ significant difference between the MAP and SAP groups in post-hoc comparison; ${ }^{\mathrm{b}}$ significant difference between the MSAP and SAP groups in post-hoc comparison; ${ }^{c}$ significant difference between the MAP and MSAP groups in post-hoc comparison. 

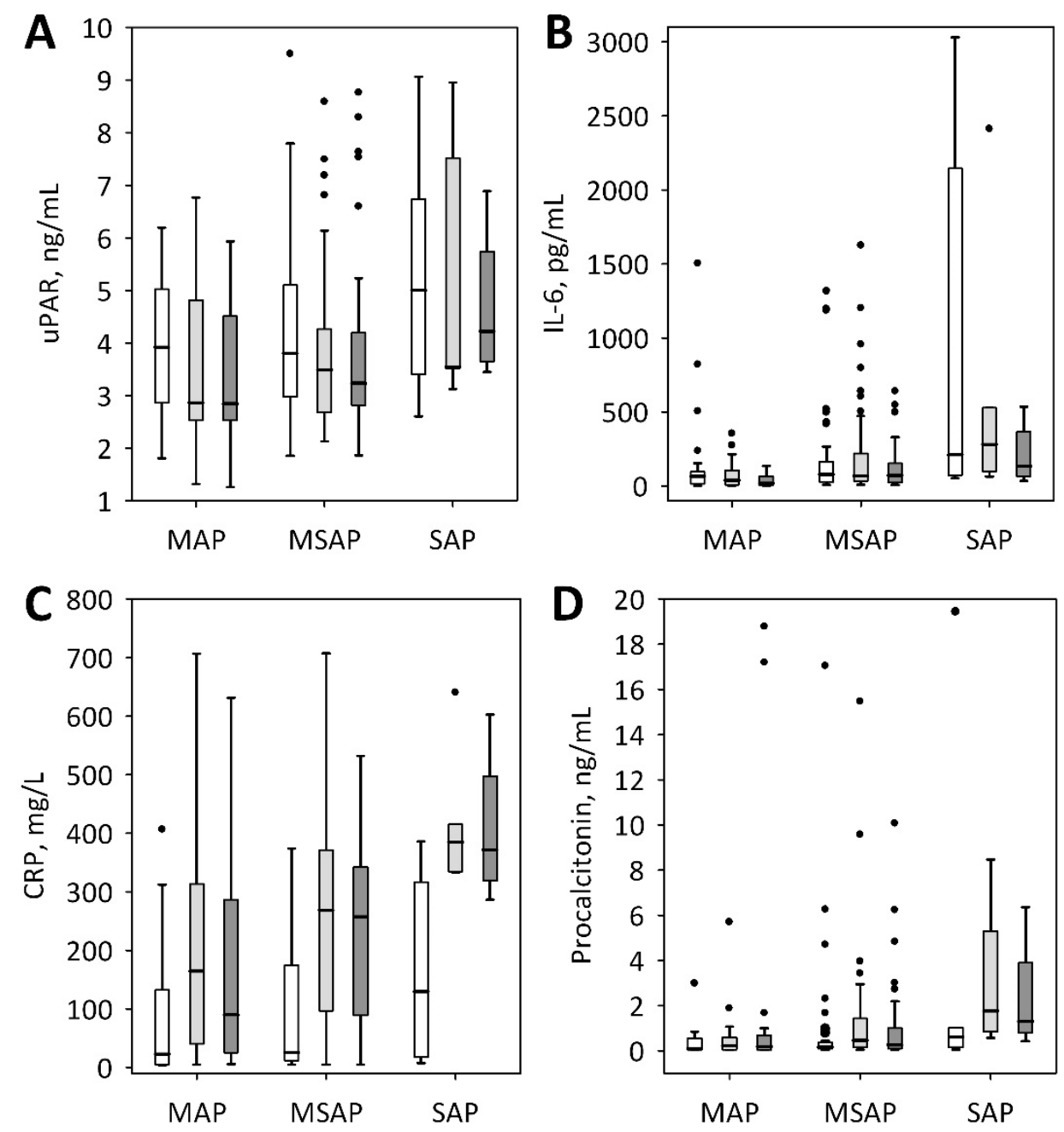

Figure 1. Serum concentrations of urokinase-type plasminogen activator receptor (UPAR) (A) among patients with mild (MAP), moderately-severe (MSAP) and severe acute pancreatitis (SAP) on admission (i.e., day 1 of the study, white bars), on day 2 (light grey bars) and day 3 (dark grey bars) of the hospital stay. Serum concentrations of interleukin-6 (IL-6) (B), C-reactive protein (CRP) (C), and procalcitonin (D) are shown for comparison. Data are presented as median, interquartile range (bars), non-outlier range (whiskers), and outliers (points).

On days 2 and 3, serum uPAR concentrations were lower as compared to the admission levels, irrespective of AP severity (Figure 2A; $p<0.001$ in MAP and MSAP groups; $p=0.039$ in SAP). This contrasted with the other inflammatory markers, i.e., IL-6 (no significant changes), CRP (significant increase on day 2 and 3 comparing to admission concentrations), and procalcitonin (increase in MSAP and SAP patients) (Figure 1B-D). Although the patients with SAP tended to present higher serum concentrations of uPAR (Figure 1A), the differences between SAP and MAP or MSAP patients were non-significant throughout the study period. Also, no significant differences were observed between patients with edematous and necrotizing pancreatitis $(p=0.8$ on day $1, p=0.6$ on day 2 and $p=0.8$ on day 3 of the study). However, serum uPAR concentrations measured on admission significantly predicted organ failure (defined as two or more points in the modified Marshall scoring system, i.e., in line with the 2012 Atlanta classification), acute cardiovascular failure, acute kidney injury, the need for intensive care, and death (Table 3). Moreover, the maximum of the three measurements of uPAR (from admission until day 3 of the hospital stay) significantly predicted the same complications (Table 3). In the multiple logistic regression, the associations between both, the admission and the maximum uPAR and organ failure, acute cardiovascular failure, acute kidney injury, ICU transfer and 
death were all significant after adjustment for sex and the presence of comorbidities. Admission uPAR was a weak predictor of Ranson's score of $\geq 3$ points (Table 3 ) and it positively correlated with the Ranson's score $(\mathrm{R}=0.27 ; p=0.012)$.
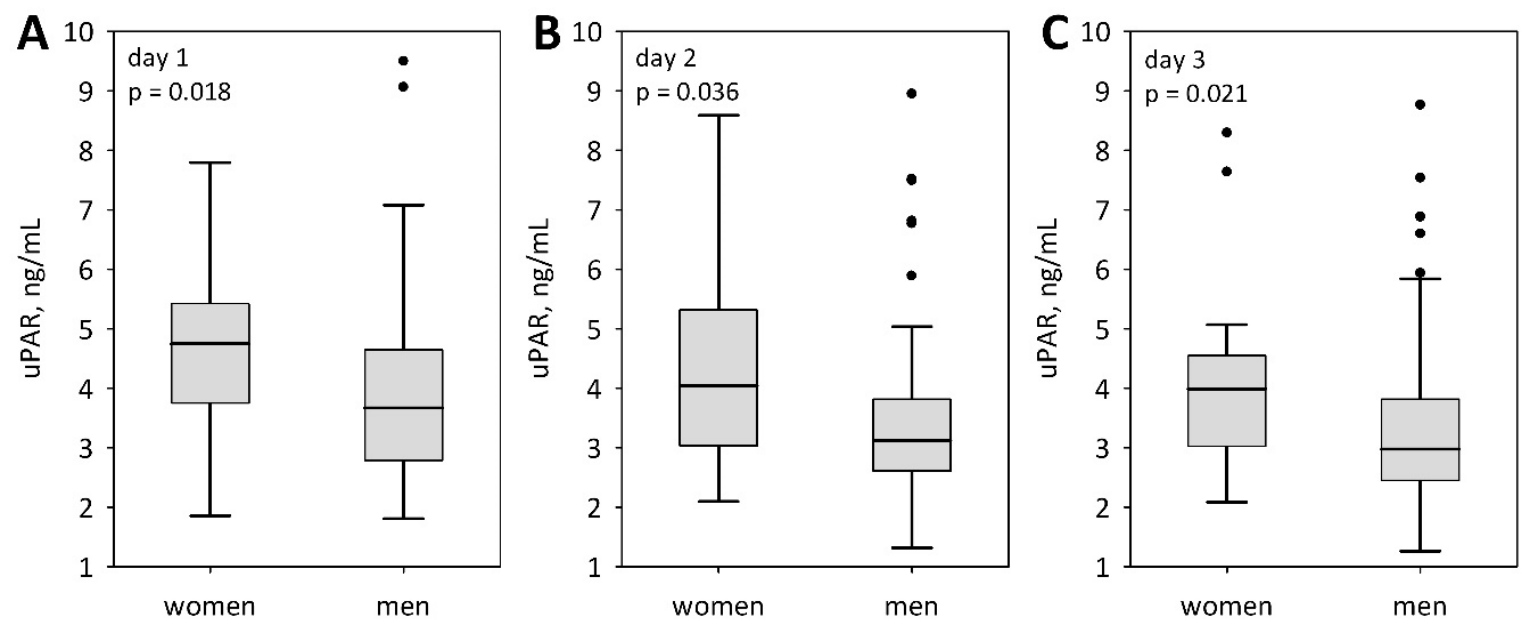

Figure 2. Sex-related differences in serum concentrations of uPAR among patients with AP during the first three days of hospital stay: On admission (day 1) (A), on day 2 (B), and on day 3 (C). Data are presented as median, interquartile range (bars), non-outlier range (whiskers), and outliers (points).

Table 3. Odds ratios (95\% confidence intervals) for serum uPAR in prediction of unfavorable course of AP.

\begin{tabular}{ccc}
\hline Dependent Variable & uPAR on Admission, per $\mathbf{1} \mathbf{~ n g} \mathbf{m L}$ & Maximum uPAR, per $\mathbf{1} \mathbf{~ n g} / \mathbf{m L}$ \\
\hline SAP (2012 Atlanta) & $1.41(0.92-2.17) ; p=0.1$ & $1.49(0.94-2.37) ; p=0.08$ \\
MSAP plus SAP (2012 Atlanta) & $1.16(0.84-1.60) ; p=0.4$ & $1.16(0.86-1.58) ; p=0.3$ \\
Persistent ( $\geq 48$ h) SIRS & $0.92(0.69-1.22) ; p=0.5$ & $0.90(0.69-1.18) ; p=0.4$ \\
Ranson $\geq$ 3 points at 48 h & $1.39(1.01-1.89) ; p=0.038$ & $1.28(0.96-1.71) ; p=0.08$ \\
Organ failure (MMSS $\geq$ 2 points) & $2.14(1.33-3.46) ; p=0.002$ & $2.06(1.30-3.26) ; p=0.002$ \\
Cardiovascular failure & $2.33(1.34-4.08) ; p=0.002$ & $2.41(1.29-4.50) ; p=0.005$ \\
ARDS & $1.01(0.59-1.72) ; p=0.9$ & $1.19(0.73-1.94) ; p=0.5$ \\
AKI & $1.78(1.11-2.84) ; p=0.015$ & $1.77(1.10-2.85) ; p=0.017$ \\
ICU transfer & $2.06(1.24-3.43) ; p=0.005$ & $2.35(1.27-4.35) ; p=0.005$ \\
Death & $1.82(1.06-3.12) ; p=0.027$ & $2.25(1.15-4.37) ; p=0.015$ \\
\hline
\end{tabular}

AKI, acute kidney injury; ARDS, acute respiratory distress syndrome; ICU, intensive care unit; MMSS, modified Marshall scoring system; MSAP, moderately severe acute pancreatitis; SAP, severe acute pancreatitis; SIRS, systemic inflammatory response syndrome; uPAR, urokinase-type plasminogen activator receptor.

We compared the diagnostic accuracy of serum uPAR for the prediction of the unfavorable course of AP with the accuracy of other proposed single markers. On admission, no significant differences were observed in the areas under the ROC curves between UPAR and IL-6, CRP, procalcitonin, D-dimer and sFlt-1 in prediction of SAP, vital organ failure and ICU transfer or death (Figure 3). Again, in this analysis, UPAR was not a significant predictor of SAP (Table 4). However, uPAR significantly predicted organ failure and ICU transfer or death. The selected cut-off values (chosen by maximizing Youden index) allowed for high specificity at relatively low sensitivity (Table 4). For comparison, the areas under the ROC curves for IL-6, CRP, procalcitonin, D-dimer and sFlt-1 are shown in Appendix A (Table A1). The diagnostic accuracy of uPAR measured on day 2 and 3, or the maximum uPAR, were not significantly better as compared to the measurements on admission, although the area under the ROC curve for uPAR measured on day 3 of the study $(72 \mathrm{~h}$ from the onset of symptoms) was significantly different from 0.5: $0.754(0.595-0.913)(p=0.002)$. 

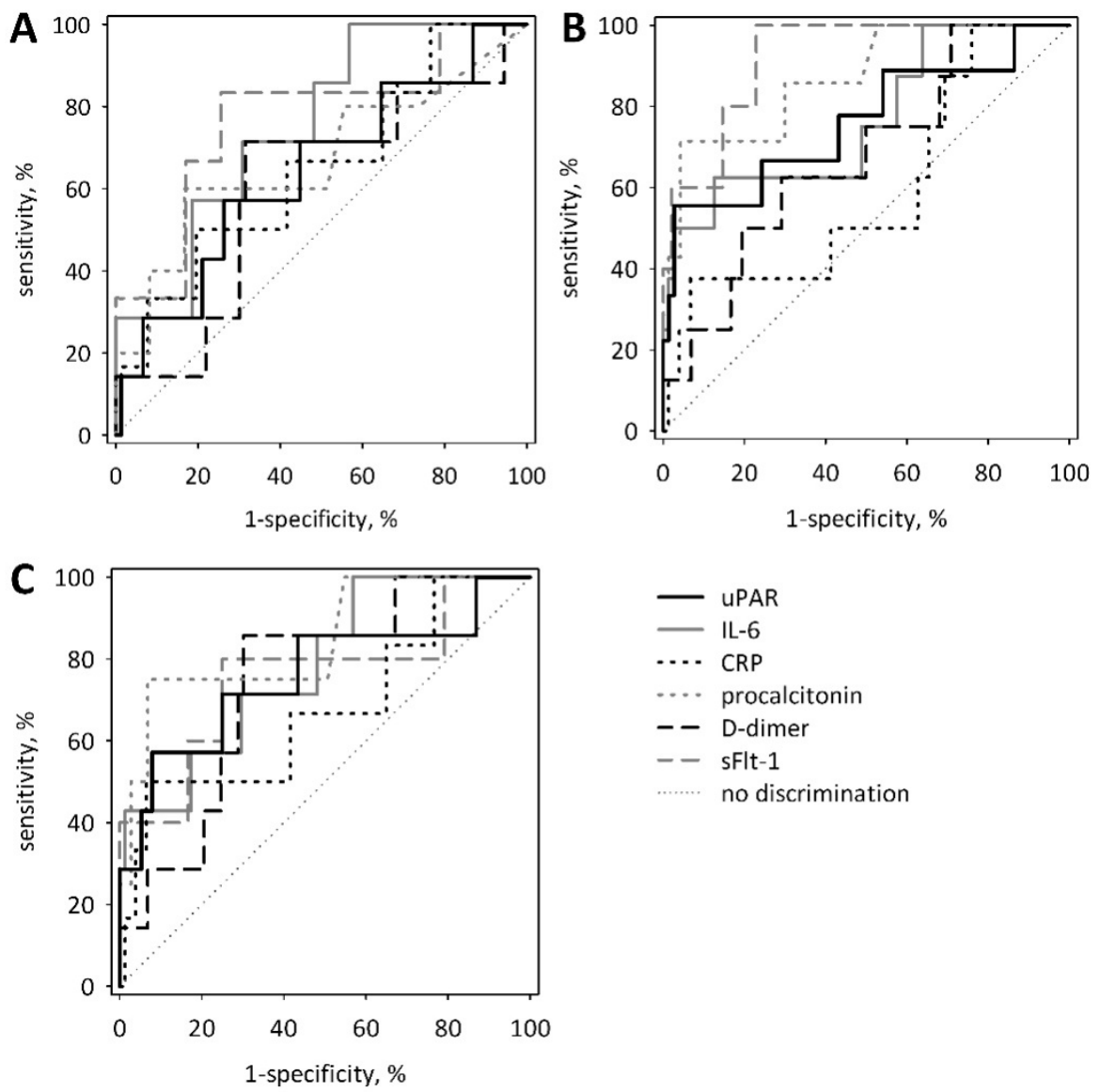

Figure 3. Receiver operating characteristic (ROC) curves for serum concentrations of uPAR on admission in prediction of SAP (A), vital organ failure (MMSS $\geq 2$ points) (B), and ICU transfer or death $(\mathbf{C})$. For comparison, ROC curves are shown for other proposed single biomarkers of AP severity measured on admission: Interleukin-6 (IL-6), C-reactive protein (CRP), procalcitonin, D-dimer, and soluble fms-like tyrosine kinase-1 (sFlt-1).

Table 4. Diagnostic accuracy of serum uPAR concentrations measured on admission for prediction of unfavorable course of AP.

\begin{tabular}{cccccc}
\hline Dependent Variable & AUC $\mathbf{( 9 5 \% ~ C I )}$ & $p^{*}$ & Cut-Off Value, $\mathbf{n g} / \mathbf{m L}$ & Sensitivity, \% & Specificity, \% \\
\hline SAP & $0.641(0.417-0.864)$ & 0.2 & 5.004 & 57 & 75 \\
Organ failure (MMSS $\geq 2$ points) & $0.761(0.565-0.958)$ & 0.009 & 6.736 & 56 & 97 \\
ICU transfer or death & $0.759(0.536-0.983)$ & 0.023 & 6.021 & 57 & 92
\end{tabular}

AUC, area under the receiver operating characteristic curve; CI, confidence interval; ICU, intensive care unit; MMSS, modified Marshall scoring system. ${ }^{*} p$-value in comparison with AUC $=0.5$.

During the studied period, uPAR concentrations correlated with the other inflammatory markers, i.e., CRP (starting from day 2 of the hospital stay), procalcitonin, and IL-6 (Table 4). Positive correlation was observed between uPAR and sFlt-1 on admission and between uPAR and D-dimer on days 1 and 3 . Serum uPAR negatively correlated with albumin (throughout the study) and hematocrit (starting from day 2). Moreover, uPAR correlated positively with bilirubin, aminotransferases and lactate dehydrogenase. No significant correlations were observed between UPAR and serum urea or creatinine concentrations (Table 5). 
Table 5. Correlations of uPAR concentrations with selected laboratory markers during early stage of acute pancreatitis.

\begin{tabular}{ccccccc}
\hline \multirow{2}{*}{ Variable } & \multicolumn{2}{c}{ Day 1 } & \multicolumn{2}{c}{ Day 2 } & \multicolumn{2}{c}{ Day 3 } \\
\cline { 2 - 7 } & $\mathbf{R}$ & $p$ & $\mathbf{R}$ & $\boldsymbol{p}$ & $\mathbf{R}$ & $p$ \\
\hline Hematocrit & -0.11 & 0.3 & -0.31 & 0.006 & -0.27 & 0.022 \\
Albumin & -0.32 & 0.014 & -0.54 & $<0.001$ & -0.44 & $<0.001$ \\
Urea & 0.16 & 0.2 & 0.10 & 0.4 & -0.04 & 0.8 \\
Creatinine & 0.18 & 0.1 & 0.05 & 0.7 & -0.05 & 0.7 \\
Bilirubin & 0.34 & 0.003 & 0.39 & $<0.001$ & 0.41 & $<0.001$ \\
AST & 0.49 & $<0.001$ & 0.53 & $<0.001$ & 0.39 & 0.001 \\
ALT & 0.37 & 0.001 & 0.37 & 0.001 & 0.30 & 0.009 \\
LDH & 0.46 & $<0.001$ & 0.28 & 0.026 & 0.52 & $<0.001$ \\
WBC & -0.03 & 0.8 & -0.03 & 0.8 & 0.19 & 0.09 \\
CRP & 0.19 & 0.1 & 0.29 & 0.012 & 0.42 & $<0.001$ \\
D-dimer & 0.26 & 0.030 & 0.19 & 0.09 & 0.30 & 0.010 \\
Procalcitonin & 0.57 & $<0.001$ & 0.54 & $<0.001$ & 0.61 & $<0.001$ \\
Interleukin 6 & 0.25 & 0.027 & 0.35 & 0.002 & 0.54 & $<0.001$ \\
sFlt-1 & 0.48 & $<0.001$ & 0.02 & 0.9 & - & - \\
\hline
\end{tabular}

ALT, alanine aminotransferase; AST, aspartate aminotransferase; CRP, C-reactive protein; LDH, lactate dehydrogenase; sFlt-1, soluble fms-like tyrosine kinase-1; uPAR, urokinase-type plasminogen activator receptor; WBC, white blood cells.

\section{Discussion}

The early identification of patients who are at risk of AP complicated by organ failure enables the provision of better care and allows for the proper allocation of intensive care resources. Despite intensive studies, we still lack laboratory markers which would allow for the early and accurate prediction of organ failure in AP. The widely adopted guidelines of the International Association of Pancreatology and the American Pancreatic Association recommend SIRS and the persistent (lasting $\geq 48 \mathrm{~h}$ ) SIRS as early markers of severe AP, however, they also acknowledge other multiparameter scores and single laboratory markers (including CRP and procalcitonin). The guidelines emphasize the need for a repeated clinical assessment which takes into account risk factors (advanced age, comorbidities, obesity), clinical signs, and response to treatment [7]. At present, no single laboratory marker can be recommended for the early prediction of AP severity.

In the current study, we evaluated the soluble UPAR, a promising biomarker previously associated with mortality and severity of acute inflammatory conditions. Although serum uPAR measured within the first $24 \mathrm{~h}$ from the onset of AP symptoms significantly predicted the complicated course of AP, including the need for ICU transfer and death, its diagnostic accuracy did not appear better than that of other inflammatory markers (IL-6, procalcitonin, CRP) nor the markers associated with endothelial dysfunction and hypercoagulability (sFlt-1, D-dimer).

The soluble UPAR is considered to be a non-specific marker of inflammation, including SIRS, both the one that is related and unrelated to infection. Yu et al. [24] and Koch et al. [20] reported positive associations between circulating UPAR and proinflammatory cytokines (IL-6, TNF- $\alpha$ ), procalcitonin and CRP. Such correlations were also observed in our study. Among patients treated in an ICU, high UPAR concentrations in serum or plasma have been associated with the severity of organ failure evaluated using the Sequential Organ Failure Assessment (SOFA), the Simplified Acute Physiology Score (SAPS) or the Acute Physiology and Chronic Health Evaluation (APACHE II) scores $[17,20,21]$. Circulating uPAR has been shown to predict mortality in patients with SIRS $[17,29,30]$, cardiovascular diseases [31-33], ARDS [34] and sepsis [22,35]. Our results also show significant associations between the serum UPAR and the subsequent organ failure, the need for ICU treatment and death. In our patients, uPAR significantly predicted AKI and cardiovascular failure, but not ARDS.

We were able to identify only two previous reports regarding circulating uPAR concentrations in AP. Nikkola et al. [36] evaluated soluble UPAR as a predictor of alcoholic AP severity. 
Excessive alcohol consumption is the cause of nearly $40 \%$ of AP cases [2], and uPAR has been previously shown to be elevated in alcoholic liver disease [37]. Nikkola et al. [36] reported significantly higher plasma uPAR among patients classified as non-mild AP (MSAP and SAP) versus those with MAP, however, they used plasma samples collected from day 1 until day 4 from admission. They estimated that uPAR at a cut-off value of $5.0 \mathrm{ng} / \mathrm{mL}$ can predict non-mild AP with the sensitivity of $79 \%$ and the specificity of $78 \%$; the area under the ROC curve was $0.81(0.70-0.92)$ [36]. This diagnostic accuracy is higher than the observed in our study, however, our data are based on results obtained on admission, within the first $24 \mathrm{~h}$ from the onset of AP symptoms. Of note, in our study uPAR did not differ between patients with AP of various etiology, in particular, no differences were observed between those with alcoholic and biliary etiology. Lipinski et al. [26] studied plasma uPAR in patients with AP of various etiologies admitted to the tertiary referral hospital. They reported a very high diagnostic accuracy of plasma uPAR for the prediction of SAP: The area under the ROC curve was $0.993(0.983-1.000)$, the diagnostic sensitivity $97 \%$ and the specificity $93 \%$ at the cut-off value of $4.75 \mathrm{ng} / \mathrm{mL}$. In addition, they reported high diagnostic accuracy of uPAR for the prediction of multiple organ failure and death: Areas under the ROC curves of 0.951 (0.951-0.991) and 0.917 (0.882-1.000), respectively [26]. Moreover, uPAR $>3.65 \mathrm{ng} / \mathrm{mL}$ allowed for the discrimination between MAP and MSAP with the $80 \%$ sensitivity and $92 \%$ specificity; the area under the ROC curve was 0.928 (0.883-0.972) [26]. The results are thus more optimistic than obtained by Nikkola et al. [36]. Obviously, our present study failed to replicate the results of Lipinski et al. [26]. There may be several reasons for this. Their study population differed from ours in the percentages of patients with SAP ( $26 \%$ versus $8 \%$ ) and MSAP ( $29 \%$ versus $61 \%$ ). The high percentage of patients with SAP in the group of Lipinski et al. [26] reflects the population of tertiary hospital patients. We can speculate that the severity of organ failure in that population may have also been higher than in our group, as the most severe cases are quickly transferred to tertiary centers. Consequently, the absolute number of patients with SAP reported by Lipinski et al. was substantially higher than in our group (33 versus 8 patients). The low number of patients with SAP in our group may in fact be responsible for the lack of a significant difference in uPAR concentrations between SAP and less severe AP, however, we also did not observe a significant difference between the MAP and MSAP patients, that was highly significant in the study of Lipinski et al. [26]. In our group, the percentage of patients with SAP is comparable to the recent reliable epidemiological data from Eastern Europe [2], but the high percentage of patients with MSAP in our group requires explanation. This is related to the arrangement of care in AP, often used in Polish secondary hospitals. We recruited patients of the surgical ward, while those with the predicted mild AP were admitted to the internal medicine/gastroenterology wards. In addition, the studies of Nikkola et al. [36], Lipinski et al. [26] and ours utilized different reagents and different samples for the measurements of uPAR: Nikkola et al. used plasma samples, but did not specify the anticoagulant. Lipinski et al. used EDTA-anticoagulated plasma, and we used serum samples. We measured uPAR with an enzyme-linked immunoassay, and although we followed good laboratory practice recommendations, the performance of the research assay could not be controlled as strictly as in the case of routine laboratory tests. Thus, the differences in laboratory methods may also be partially responsible for the differences in results of the three studies. This implicates the need for standardization of the laboratory methods before wider clinical use of UPAR.

In our study, uPAR correlated significantly with the laboratory markers of liver injury (bilirubin, transaminanases). Previous reports in AP did not explore such correlations, however, similar relationships were observed in the critically ill [20]. Koch et al. [20] observed significant correlations between uPAR and the markers of renal function, not present in our study. However, in our patients, high UPAR was a predictor of the subsequent AKI. Moreover, we studied the associations between the demographic variables and UPAR and observed significantly higher concentrations in women. This is in line with previous reports, including the study of Nikkola et al. [36] in alcoholic AP, but also the studies regarding other groups of patients [38,39]. 
The main and the most important limitation of our study is the low number of patients with SAP. Moreover, for the above-mentioned reasons, the percentage of patients with MAP and MSAP differ from the general epidemiological data. Therefore, we cannot draw definitive conclusions. Nonetheless, our study suggests that circulating uPAR measured on admission to a secondary care hospital allows for the prediction of the complicated course of AP with moderate diagnostic accuracy, comparable to other inflammatory, coagulation or endothelial markers, including the ones widely available in the clinical practice (CRP, procalcitonin, and D-dimer). Thus, it is too early to advocate for the use of uPAR in the early assessment of AP severity. More studies are needed that would allow the evaluation of the robustness of uPAR diagnostic performance in other health care settings. Also, the lack of laboratory standardization of uPAR measurements must be taken into account before wider clinical use of the marker.

Author Contributions: Conceptualization: W.K., B.K.-C., P.C. and M.P.; methodology: W.K., P.D., M.M.-L. and B.K.-C.; software: P.D.; validation: W.K., P.D., P.C. and B.K.-C.; formal analysis: B.K.-C., P.D. and P.C.; investigation: W.K., B.K.-C. and M.M.-L.; resources: W.K.; data curation: W.K. and B.K.-C.; writing-original draft preparation: W.K., B.K.-C. and P.C.; writing—review and editing: P.D., B.K.-C., M.P., M.M. and P.C.; visualization: P.D. and M.M.; supervision: B.K.-C.; project administration: B.K.-C. and P.C.; funding acquisition: W.K. and B.K.-C. Witold Kolber and Beata Kuśnierz-Cabala have contributed equally to this work.

Funding: This work was partly supported by grant K/ZDS/006347 from Jagiellonian University Medical College.

Acknowledgments: The authors thank Marek Kuźniewski and Jerzy Walocha for their support and inspiring discussions during the preparation of the manuscript. We acknowledge Tomasz Kasprzycki for help in language corrections.

Conflicts of Interest: The authors declare that they have no conflict of interest.

\section{Appendix}

Table A1. The values of area under the receiver operating characteristic curve (AUC) with $95 \%$ confidence intervals (95\% CI) for single laboratory markers proposed for the early assessment of severity of acute pancreatitis that were measured in the study and compared with uPAR. We provide AUC values estimated based on results obtained on the day of admission.

\begin{tabular}{cccc}
\hline \multirow{2}{*}{ Laboratory Marker } & \multicolumn{3}{c}{ AUC (95\% CI); $p$-Value * for Prediction of } \\
\cline { 2 - 4 } & SAP & Organ Failure (MMSS $\geq \mathbf{2}$ Points) & ICU Transfer or Death \\
\hline IL-6 & $0.753(0.590-0.917) ; p=0.002$ & $0.767(0.578-0.956) ; p=0.006$ & $0.781(0.610-0.953) ; p=0.001$ \\
CRP & $0.647(0.412-0.882) ; p=0.2$ & $0.592(0.373-0.810) ; p=0.4$ & $0.675(0.425-0.925) ; p=0.2$ \\
Procalcitonin & $0.669(0.378-0.961) ; p=0.3$ & $0.870(0.729-1.000) ; p<0.001$ & $0.844(0.627-1.000) ; p=0.002$ \\
D-dimer & $0.605(0.377-0.901) ; p=0.4$ & $0.674(0.485-0.863) ; p=0.07$ & $0.746(0.582-0.909) ; p=0.003$ \\
sFlt-1 & $0.770(0.546-0.993) ; p=0.018$ & $0.921(0.825-1.000) ; p<0.001$ & $0.758(0.495-1.000) ; p=0.054$ \\
\hline \multicolumn{5}{c}{$* p$-value in comparison with AUC $=0.5}$.
\end{tabular}

\section{References}

1. Baron, T. Managing severe acute pancreatitis. Clevel. Clin. J. Med. 2013, 80, 354-359. [CrossRef] [PubMed]

2. Párniczky, A.; Kui, B.; Szentesi, A.; Balázs, A.; Szúcs, Á.; Mosztbacher, D.; Czimmer, J.; Sarlós, P.; Bajor, J.; Gódi, S.; et al. Prospective, multicentre, nationwide clinical data from 600 cases of acute pancreatitis. PLoS ONE 2016, 11, e0165309. [CrossRef] [PubMed]

3. Wu, B.U.; Banks, P. Clinical management of patients with acute pancreatitis. Gastroenterology 2013, 144, 1272-1281. [CrossRef] [PubMed]

4. Tenner, S.; Baillie, J.; Dewitt, J.; Vege, S.S. American College of Gastroenterology guideline: Management of acute pancreatitis. Am. J. Gastroenterol. 2013, 108, 1400-1415. [CrossRef] [PubMed]

5. Wereszczyńska-Siemiątkowska, U.; Siemiątkowki, A. Mediators of inflammatory and coagulation processes in acute pancreatitis-Selected issues. Postępy Nauk Med. 2011, 3, 222-231. 
6. Banks, P.A.; Bollen, T.L.; Dervenis, C.; Gooszen, H.G.; Johnson, C.D.; Sarr, M.G.; Tsiotos, G.C.; Vege, S.S. Classification of acute pancreatitis-2012: Revision of the Atlanta classification and definitions by international consensus. Gut 2013, 62, 102-111. [CrossRef] [PubMed]

7. Working Group IAP/APA Acute Pancreatitis Guidelines. IAP/APA evidence-based guidelines for the management of acute pancreatitis. Pancreatology 2013, 13, e1-e15. [CrossRef] [PubMed]

8. Swaroop, S.S.; Whitcomb, D.C.; Grover, S. Predicting the Severity of Acute Pancreatitis. UpToDate. Available online: http:/ / www.uptodate.com (accessed on 12 April 2018).

9. Naskalski, J.W.; Kusnierz-Cabala, B.; Panek, J.; Kedra, B. Poly-C specific ribonuclease activity correlates with increased concentrations of IL-6, IL-8 and sTNFR55/TNFR75 in plasma of patients with acute pancreatitis. J. Physiol. Pharmacol. 2003, 54, 439-448. [PubMed]

10. Gurda-Duda, A.; Kusnierz-Cabala, B.; Nowak, W.; Naskalski, J.W.; Kulig, J. Assessment of prognostic value of certain acute phase proteins and procalcitonin in the prognosis of acute pancreatitis. Pancreas 2008, 37, 449-453. [CrossRef] [PubMed]

11. Kuśnierz-Cabala, B.; Nowak, E.; Sporek, M.; Kowalik, A.; Kuźniewski, M.; Enguita, F.J.; Stępień, E. Serum levels of unique miR-551-5p and endothelial-specific miR-126a-5p allow discrimination of patients in the early phase of acute pancreatitis. Pancreatology 2015, 15, 344-351. [CrossRef] [PubMed]

12. Spitzer, A.L.; Barcia, A.M.; Schell, M.T.; Barber, A.; Norman, J.; Grendell, J.; Harris, H.W. Applying Ockham's Razor to pancreatitis prognostication. Ann. Surg. 2006, 243, 380-388. [CrossRef] [PubMed]

13. Huang, H.L.; Nie, X.; Cai, B.; He, Y.; Miao, Q.; Song, H.L.; Luo, T.X.; Gao, B.X.; Wang, L.L.; Li, G.X. Procalcitonin levels predicts acute kidney injury and prognosis in acute pancreatitis: A prospective study. PLoS ONE 2013, 8, e82250. [CrossRef] [PubMed]

14. Dumnicka, P.; Maduzia, D.; Ceranowicz, P.; Olszanecki, R.; Drożdż, R.; Kuśnierz-Cabala, B. The interplay between inflammation, coagulation and endothelial injury in the early phase of acute pancreatitis: Clinical implications. Int. J. Mol. Sci. 2017, 18, 354. [CrossRef] [PubMed]

15. Dumnicka, P.; Sporek, M.; Mazur-Laskowska, M.; Ceranowicz, P.; Kuźniewski, M.; Drożdż, R.; Ambroży, T.; Olszanecki, R.; Kuśnierz-Cabala, B. Serum soluble Fms-like tyrosine kinase 1 (sFlt-1) predicts the severity of acute pancreatitis. Int. J. Mol. Sci. 2017, 17, 2038. [CrossRef] [PubMed]

16. Dumnicka, P.; Kuśnierz-Cabala, B.; Sporek, M.; Mazur-Laskowska, M.; Gil, K.; Kuźniewski, M.; Ceranowicz, P.; Warzecha, Z.; Dembiński, A.; Bonior, J.; et al. Serum concentrations of Angiopoietin-2 and soluble fms-like tyrosine kinase (sFlt-1) are associated with coagulopathy among patients with acute pancreatitis. Int. J. Mol. Sci. 2017, 18, 753. [CrossRef] [PubMed]

17. Wu, X.; Long, D.; Yu, L.; Yang, J.H.; Zhang, Y.C.; Geng, F. Urokinase-type plasminogen activator receptor as a predictor of poor outcome in patients with systemic inflammatory response syndrome. World J. Emerg. Med. 2013, 4, 190-195. [CrossRef] [PubMed]

18. Iba, T.; Gando, S.; Murata, A.; Kushimoto, S.; Saitoh, D.; Eguchi, Y.; Ohtomo, Y.; Okamoto, K.; Koseki, K.; Mayumi, T.; et al. Predicting the severity of Systemic Inflammatory Response Syndrome (SIRS)-associated coagulopathy with hemostatic molecular markers and vascular endothelial injury markers. J. Trauma 2007, 63, 1093-1098. [CrossRef] [PubMed]

19. Friess, H.; Duarte, R.; Kleeff, J.; Fukuda, A.; Tang, W.H.; Graber, H.; Schilling, M.; Zimmermann, A.; Korc, M.; Büchler, M.W. The plasminogen activator/plasmin system is up-regulated after acute necrotizing pancreatitis in human beings. Surgery 1998, 124, 79-86. [CrossRef]

20. Koch, A.; Voigt, S.; Kruschinski, C.; Sanson, E.; Duckers, H.; Horn, A.; Yagmur, E.; Zimmermann, H.; Trautwein, C.; Tacke, F. Circulating soluble urokinase plasminogen activator receptor is stably elevated during the first week of treatment in the intensive care unit and predicts mortality in critically ill patients. Crit. Care 2011, 15, R63. [CrossRef] [PubMed]

21. Backes, Y.; Sluijs, K.F.; Mackie, D.P.; Tacke, F.; Koch, A.; Teuhunen, J.J.; Schultz, M.J. Usefulness of suPAR as a biological marker in patients with systemic inflammation or infection: A systemic review. Intensive Care Med. 2012, 38, 1418-1428. [CrossRef] [PubMed]

22. Donadello, K.; Scalletta, S.; Covajes, C.; Vincent, J.L. suPAR as a prognostic biomarker in sepsis. BMC Med. 2012, 10, 2. [CrossRef] [PubMed]

23. Thunø, M.; Macho, B.; Eugen-Olsen, J. suPAR: The molecular crystal ball. Dis. Mark. 2009, 27, $157-172$. [CrossRef] 
24. Yu, L.; Long, D.; Wu, X.L.; Yang, J.H.; Yang, Y.C.; Feng, G. Prognostic significance of urokinase-type plasminogen activator and its receptor in patients with systemic inflammatory response syndrome. World J. Emerg. Med. 2011, 2, 185-189. [CrossRef] [PubMed]

25. Seppala, U.; Huttunen, R.; Tarkka, M.; Aittoniemi, J.; Koskinen, J.; Leino, A.; Vahlberg, T.; Rintala, E.M. Soluble urokinase-type plasminogen activator in patients with suspected infection in the emergency room: A prospective cohort study. J. Intern. Med. 2012, 272, 247-256. [CrossRef] [PubMed]

26. Lipinski, M.; Rydzewska-Rosołowska, A.; Rydzewski, A.; Cicha, M. Rydzewska G: Soluble urokinase-type activator receptor (suPAR) patients with acute pancreatitis (AP)-Progress in prediction of AP severity. Pancreatology 2017, 17, 24-29. [CrossRef] [PubMed]

27. Kellum, J.A.; Lameire, N.; Aspelin, P.; Barsoum, R.S.; Burdmann, E.A.; Goldstein, S.L.; Herzog, C.A.; Joannidis, M.; Kribben, A.; Levey, A.S.; et al. Kidney Disease: Improving Global Outcomes (KDIGO) Acute Kidney Injury Work Group. KDIGO clinical practice guideline for acute kidney injury. Kidney Int. Suppl. 2012, 2, 1-138.

28. ARDS Definition Task Force; Ranieri, V.M.; Rubenfeld, G.D.; Thompson, B.T.; Ferguson, N.D.; Caldwell, E.; Fan, E.; Camporota, L.; Slutsky, A.S. Acute respiratory distress syndrome: The Berlin Definition. JAMA 2012, 307, 2526-2533. [PubMed]

29. Kofoed, K.; Eugen-Olsen, J.; Petersen, J.; Larsen, K.; Anderson, O. Predicting mortality in patients with systemic inflammatory response syndrome: An evaluation of two prognostic models, two soluble receptors, and a macrophage migration inhibitory factor. Eur. J. Clin. Microbiol. Infect. Dis. 2008, 27, 375-383. [CrossRef] [PubMed]

30. Raggam, R.B.; Wagner, J.; Prüller, F.; Grisold, A.; Leitner, E.; Zollner-Schwetz, I.; Valentin, T.; Krause, R.; Hoenigl, M. Soluble urokinase plasminogen activator receptor predicts mortality in patients with systemic inflammatory response syndrome. J. Intern. Med. 2014, 276, 651-658. [CrossRef] [PubMed]

31. Botha, S.; Fourie, C.M.; Schutte, R.; Eugen-Olsen, J.; Schutte, A.E. Soluble urokinase plasminogen activator receptor as a prognostic marker of all-cause and cardiovascular mortality in black population. Int. J. Cardiol. 2015, 184, 631-636. [CrossRef] [PubMed]

32. Koller, L.; Stojkovic, S.; Richter, B.; Sulzgruber, P.; Potolidis, C.; Liebhart, F.; Mörtl, D.; Berger, R.; Goliasch, G.; Wojta, J.; et al. Soluble urokinase-type plasminogen activator receptor improves risk prediction in patients with chronic heart failure. JACC Heart Fail. 2017, 5, 268-277. [CrossRef] [PubMed]

33. Lyngbæk, S.; Andersson, C.; Marott, J.L.; Møller, D.V.; Christiansen, M.; Iversen, K.K.; Clemmensen, P.; Eugen-Olsen, J.; Hansen, P.R.; Jeppesen, J.L. Soluble urokinase plasminogen activator receptor for risk prediction in patients admitted with acute chest pain. Clin. Chem. 2013, 59, 1621-1629. [CrossRef] [PubMed]

34. Geboers, D.; de Beer, F.; Tuip-de Boer, A.; van der Poll, T.; Horn, J.; Cremer, O.; Bonten, M.J.; Ong, D.S.; Schultz, M.J.; Bos, L.D. Plasma suPAR as a prognostic biological marker for ICU mortality in ARDS patients. Int. Care Med. 2015, 41, 1281-1290. [CrossRef] [PubMed]

35. Giamarellos-Bourboulis, E.J.; Norrby-Teglund, A.; Mylona, V.; Savva, A.; Tsangaris, I.; Dimopoulou, I.; Mouktaroudi, M.; Raftogiannis, M.; Georgitsi, M.; Linnér, A.; et al. Risk assessment in sepsis: A new prognostication rule by APACHE II score and serum soluble urokinase plasminogen activator receptor. Crit. Care 2012, 16, R149. [CrossRef] [PubMed]

36. Nikkola, A.; Aittoniemi, J.; Huttunen, R.; Rajala, L.; Nordback, I.; Sand, J.; Laukkarinen, J. Plasma level of soluble urokinase-type plasminogen activator receptor predicts the severity of acute alcohol pancreatitis. Pancreas 2017, 46, 77-82. [CrossRef] [PubMed]

37. Zimmermann, H.W.; Koch, A.; Seidler, S.; Trautwein, C.; Tacke, F. Circulating soluble urokinase plasminogen activator is elevated in patients with chronic liver disease, discriminates stage and aetiology of cirrhosis and predicts prognosis. Liver Int. 2011, 32, 500-509. [CrossRef] [PubMed] 
38. Wei, C.; Trachtman, H.; Li, J.; Dong, C.; Friedman, A.L.; Gassman, J.J.; McMahan, J.L.; Radeva, M.; Heil, K.M.; Trautmann, A.; et al. Circulating suPAR in two cohorts of primary FSGS. J. Am. Soc. Nephrol. 2012, 23, 2051-2059. [CrossRef] [PubMed]

39. Lyngbæk, S.; Marott, J.L.; Møller, D.V.; Christiansen, M.; Iversen, K.K.; Clemmensen, P.M.; Eugen-Olsen, J.; Jeppesen, J.L.; Hansen, P.R. Usefulness of soluble urokinase plasminogen activator receptor to predict repeat myocardial infarction and mortality in patients with ST-segment elevation myocardial infarction undergoing primary percutaneous intervention. Am. J. Cardiol. 2012, 110, 1756-1763. [CrossRef] [PubMed]

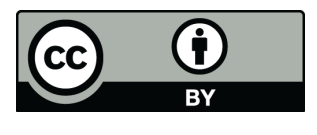

(C) 2018 by the authors. Licensee MDPI, Basel, Switzerland. This article is an open access article distributed under the terms and conditions of the Creative Commons Attribution (CC BY) license (http:/ / creativecommons.org/licenses/by/4.0/). 